\title{
NOTES ON FORMAL MANIPULATIONS OF DOUBLE SERIES
}

\author{
JunESANG ChOI
}

\begin{abstract}
A BSTRACT. Formal manipulations of double series are useful in getting some other identities from given ones and evaluating certain summations, involving double series. The main object of this note is to summarize rather useful double series manipulations scattered in the literature and give their generalized formulas, for convenience and easier reference in their future use.

An application of such manipulations to an evaluation for Euler sums (in itself, interesting), among other things, will also be presented to show usefulness of such manipulative techniques.
\end{abstract}

\section{Introduction and formulas}

We first give generalized formulas of some known identities of formal manipulations, without thinking of their convergence, of double series scattered in the literature. Such double series identities as those given here may be considered purely formal, yet we shall find that the manipulative techniques are fully as useful as when applied to convergent series.

Those formulas given here are useful, among other things, in the study of generating functions of sets of polynomials which are appeared in the theory of special functions ( $c f$. Rainville [5]). Schwatt [6, Chapter 1] also made extensive use of those special cases of manipulative techniques given here in dealing with double series involving elementary functions. To show further usefulness of the principles given here, we next apply one of these double series manipulations to evaluate an Euler sum by following Nielsen's work [4] in an easier and a little more detailed way.

Received May 3, 2002.

2000 Mathematics Subject Classification: Primary 33-00, 40A25; Secondary 11L99.

Key words and phrases: double series manipulations, Riemann Zeta function, generalized harmonic numbers, harmonic numbers, Euler sums.

This work is supported by the Dongguk University Research Fund. 
Now we give some generalized principles of double series manipulations some special cases of which are also written for easy reference in their use. Note that $A_{x, y}$ denotes a function of two variables $x$ and $y$, and $\mathbb{N}$ the set of positive integers in what follows.

$$
\sum_{n=0}^{\infty} \sum_{k=0}^{\infty} A_{k, n}=\sum_{n=0}^{\infty} \sum_{k=0}^{[n / p]} A_{k, n-p k} \quad(p \in \mathbb{N}),
$$

which may be written in reverse order

$$
\begin{aligned}
& \sum_{n=0}^{\infty} \sum_{k=0}^{[n / p]} A_{k, n}=\sum_{n=0}^{\infty} \sum_{k=0}^{\infty} A_{k, n+p k} \quad(p \in \mathbb{N}) . \\
& \sum_{n=0}^{\infty} \sum_{k=0}^{n} A_{k, n}=\sum_{n=0}^{\infty} \sum_{k=0}^{[n / p]} A_{k, n-(p-1) k} \quad(p-1 \in \mathbb{N}) .
\end{aligned}
$$

Some special cases (1.4) through (1.8) of these three principles are written here:

$$
\begin{aligned}
& \sum_{n=0}^{\infty} \sum_{k=0}^{\infty} A_{k, n}=\sum_{n=0}^{\infty} \sum_{k=0}^{n} A_{k, n-k} ; \\
& \sum_{n=0}^{\infty} \sum_{k=0}^{n} A_{k, n}=\sum_{n=0}^{\infty} \sum_{k=0}^{\infty} A_{k, n+k} ; \\
& \sum_{n=0}^{\infty} \sum_{k=0}^{\infty} A_{k, n}=\sum_{n=0}^{\infty} \sum_{k=0}^{[n / 2]} A_{k, n-2 k} ; \\
& \sum_{n=0}^{\infty} \sum_{k=0}^{[n / 2]} A_{k, n}=\sum_{n=0}^{\infty} \sum_{k=0}^{\infty} A_{k, n+2 k} ; \\
& \sum_{n=0}^{\infty} \sum_{k=0}^{n} A_{k, n}=\sum_{n=0}^{\infty} \sum_{k=0}^{[n / 2]} A_{k, n-k} .
\end{aligned}
$$




$$
\sum_{\ell=0}^{n} \sum_{k=\ell}^{p \ell} A_{\ell, k}=\sum_{k=0}^{p n} \sum_{\ell=\left[\frac{k+p-1}{p}\right]}^{k} A_{\ell, k} \quad(p \in \mathbb{N})
$$

which can be proved as follows: Expanding the first member, we obtain

$$
\sum_{k=0}^{0} A_{0, k}+\sum_{k=1}^{p} A_{1, k}+\cdots+\sum_{k=n}^{p n} A_{n, k} .
$$

Writing the terms with equal indices of $k$ in columns and adding these columns yields the desired identity. Likewise, other principles may also be proved. For another possible way of proof, see Choi and Seo [2] and Rainville [5].

Similarly as in (1.9), we have

$$
\sum_{n=0}^{\infty} \sum_{k=n}^{p n} A_{n, k}=\sum_{k=0}^{\infty} \sum_{n=\left[\frac{k+p-1}{p}\right]}^{k} A_{n, k} \quad(p \in \mathbb{N}) .
$$

Some special cases (1.11) through (1.14) of (1.9) and (1.10) are, for easier reference, written:

$$
\begin{aligned}
& \sum_{\ell=0}^{n} \sum_{k=\ell}^{2 \ell} A_{\ell, k}=\sum_{k=0}^{2 n} \sum_{\ell=\left[\frac{k+1}{2}\right]}^{k} A_{\ell, k} ; \\
& \sum_{\ell=0}^{n} \sum_{k=\ell}^{3 \ell} A_{\ell, k}=\sum_{k=0}^{3 n} \sum_{\ell=\left[\frac{k+2}{3}\right]}^{k} A_{\ell, k} . \\
& \sum_{n=0}^{\infty} \sum_{k=n}^{2 n} A_{n, k}=\sum_{k=0}^{\infty} \sum_{n=\left[\frac{k+1}{2}\right]}^{k} A_{n, k}
\end{aligned}
$$

and

$$
\sum_{n=0}^{\infty} \sum_{k=n}^{3 n} A_{n, k}=\sum_{k=0}^{\infty} \sum_{n=\left[\frac{k+2}{3}\right]}^{k} A_{n, k}
$$




$$
\sum_{k=\ell}^{\infty} \sum_{n=k+(p-1) \ell}^{\infty} A_{k, n}=\sum_{n=p \ell}^{\infty} \sum_{k=\ell}^{n-(p-1) \ell} A_{k, n} \quad(p-1 \in \mathbb{N} ; \ell+1 \in \mathbb{N}) .
$$

Setting $\mathrm{p}=2$ in (1.15), we get

$$
\sum_{k=\ell}^{\infty} \sum_{n=k+\ell}^{\infty} A_{k, n}=\sum_{n=2 \ell}^{\infty} \sum_{k=\ell}^{n-\ell} A_{k, n} \quad(\ell+1 \in \mathbb{N}),
$$

which, for $\ell=0$ and $\ell=1$, yields

$$
\begin{aligned}
\sum_{k=0}^{\infty} \sum_{n=k}^{\infty} A_{k, n} & =\sum_{n=0}^{\infty} \sum_{k=0}^{n} A_{k, n} \\
\sum_{k=1}^{\infty} \sum_{n=k+1}^{\infty} A_{k, n} & =\sum_{n=2}^{\infty} \sum_{k=1}^{n-1} A_{k, n}
\end{aligned}
$$

which will be used in Section 2.

$$
\sum_{k=1}^{\infty} \sum_{n=k}^{p k} A_{k, n}=\sum_{n=1}^{\infty} \sum_{k=0}^{[(p-1) n / p]} A_{n-k, n} \quad(p-1 \in \mathbb{N}),
$$

which, for $p=2$, becomes

$$
\sum_{k=1}^{\infty} \sum_{n=k}^{2 k} A_{k, n}=\sum_{n=1}^{\infty} \sum_{k=0}^{[n / 2]} A_{n-k, n}
$$

$$
\sum_{n=1}^{\infty} \sum_{k=q}^{p n} A_{n, k}=\sum_{k=q}^{\infty} \sum_{n=\left[\frac{k+p-1}{p}\right]}^{\infty} A_{n, k} \quad(p-1 \in \mathbb{N} ; q-1 \in \mathbb{N} ; q \leq p),
$$

which, for $p=2$ and $q=2$, yields

$$
\sum_{n=1}^{\infty} \sum_{k=2}^{2 n} A_{n, k}=\sum_{k=2}^{\infty} \sum_{n=\left[\frac{k+1}{2}\right]}^{\infty} A_{n, k}
$$




$$
\sum_{k=1}^{n} \sum_{\ell=1}^{k} A_{k, \ell}=\sum_{\ell=1}^{n} \sum_{k=\ell}^{n} A_{k, \ell}
$$

in which starting points may be changed as follows:

$$
\sum_{k=0}^{n} \sum_{\ell=0}^{k} A_{k, \ell}=\sum_{\ell=0}^{n} \sum_{k=\ell}^{n} A_{k, \ell}
$$

which is also a useful formula (see Choi [3, p. 671, Eq. (2.5)]).

It is remarked that there is no bound to the number of such identities. Whenever necessary, some desired principles can be made.

\section{An application}

Those formulas given in Section 1 have been used in various ways. Here we apply one of them to evaluate a sum involving harmonic numbers, known as Euler Sums, the method of which was originally due to Euler who used divergent series in his evaluation (see Borwein et al. [1]). On the other hand, Nielsen used Euler's decomposition formula and one of those formulas in Section 2 implicitly (to avoid using divergent series in Euler's evaluation) to get a more general formula containing the socalled Euler formula for harmonic sums as a special case (see Nielsen [4, Kapitel III]).

The Riemann Zeta function $\zeta(s)$ is defined by

$$
\zeta(s):=\sum_{n=1}^{\infty} \frac{1}{n^{s}} \quad(\Re(s)>1),
$$

which can be continued meromorphically to the whole complex $s$-plane (except for a simple pole at $s=1$ with its residue 1 ) by means of the contour integral representation (see Srivastava and Choi $[8$, p. 97, Eq.(5)]) or various other integral representations (see Srivastava and Choi [8, pp. 100-103]).

When $c_{n, p}$ is defined by

$$
c_{n, p}:=\sum_{r=1}^{\infty} \frac{H_{r}^{(p)}}{(r+1)^{n}} \quad(n-1 \in \mathbb{N} ; p \in \mathbb{N}),
$$


where $H_{r}^{(p)}$ denotes the generalized harmonic numbers defined by

$$
H_{r}^{(p)}:=\sum_{j=1}^{r} \frac{1}{j^{p}} \quad(k, p \in \mathbb{N}),
$$

and the harmonic number $H_{r}$ :

$$
H_{r}:=H_{r}^{(1)}=\sum_{j=1}^{r} \frac{1}{j},
$$

we have a well-known reflection formula:

(5) $\quad \zeta(n) \zeta(p)=\zeta(n+p)+c_{n, p}+c_{p, n} \quad(n-1 \in \mathbb{N} ; p-1 \in \mathbb{N})$.

Indeed, by using (1.18) for the fourth equality,

$$
\begin{aligned}
\zeta(n) \zeta(p) & =\sum_{k=1}^{\infty} \sum_{j=1}^{\infty} \frac{1}{k^{n} j^{p}} \\
& =\sum_{k=1}^{\infty} \frac{1}{k^{n}}\left(\frac{1}{k^{p}}+\sum_{j=1}^{k-1} \frac{1}{j^{p}}+\sum_{j=k+1}^{\infty} \frac{1}{j^{p}}\right) \\
& =\sum_{k=1}^{\infty} \frac{1}{k^{n+p}}+\sum_{k=1}^{\infty} \frac{1}{k^{n}}\left(\sum_{j=1}^{k-1} \frac{1}{j^{p}}\right)+\sum_{k=1}^{\infty} \sum_{j=k+1}^{\infty} \frac{1}{k^{n} j^{p}} \\
& =\zeta(n+p)+c_{n, p}+\sum_{j=2}^{\infty} \frac{1}{j^{p}}\left(\sum_{k=1}^{j-1} \frac{1}{k^{n}}\right) \\
& =\zeta(n+p)+c_{n, p}+c_{p, n} .
\end{aligned}
$$

We recall here a known decomposition formula due, originally, to Euler (see Nielsen [4, p. 48, Eq. (9)]):

(6)

$$
\begin{aligned}
\frac{1}{x^{p}(x-a)^{q}}= & (-1)^{q} \sum_{r=0}^{p-1}\left(\begin{array}{c}
q+\dot{r}-1 \\
q-1
\end{array}\right) \frac{1}{x^{p-r} a^{q+r}} \\
& +\sum_{r=0}^{q-1}\left(\begin{array}{c}
p+r-1 \\
p-1
\end{array}\right) \frac{(-1)^{r}}{a^{p+r}(x-a)^{q-r}} \quad(p \in \mathbb{N} ; q \in \mathbb{N}),
\end{aligned}
$$


the special case of which when $a=1$ is recorded in the work of Borwein et al. [1, p. 284] who used it to evaluate Euler sums by using matrix manipulation.

Setting $x=n$ in (6) and summing the resulting equation from $a=1$ to $a=n-1$, we obtain

$$
\sum_{a=1}^{n-1} \frac{1}{n^{p} a^{q}}=A_{n}+B_{n},
$$

where $A_{n}$ and $B_{n}$ are given by

$$
A_{n}=(-1)^{q} \sum_{r=0}^{p-1}\left(\begin{array}{c}
q+r-1 \\
q-1
\end{array}\right) \sum_{a=1}^{n-1} \frac{1}{n^{p-r} a^{q+r}}
$$

and

$$
B_{n}=\sum_{r=0}^{q-1}(-1)^{r}\left(\begin{array}{c}
p+r-1 \\
p-1
\end{array}\right) \sum_{a=1}^{n-1} \frac{1}{a^{p+r}(n-a)^{q-r}} .
$$

We then find that

$$
\begin{aligned}
A_{n}+B_{n}= & (-1)^{q} \sum_{r=0}^{p-2}\left(\begin{array}{c}
q+r-1 \\
q-1
\end{array}\right) \sum_{a=1}^{n-1} \frac{1}{n^{p-r} a^{q+r}} \\
& +\sum_{r=0}^{q-2}(-1)^{r}\left(\begin{array}{c}
p+r-1 \\
p-1
\end{array}\right) \sum_{a=1}^{n-1} \frac{1}{a^{p+r}(n-a)^{q-r}}+C_{n}
\end{aligned}
$$

where

$$
C_{n}:=-(-1)^{q}\left(\begin{array}{c}
p+q-2 \\
p-1
\end{array}\right) \sum_{a=1}^{n-1} \frac{1}{a^{p+q-2} n(n-a)} .
$$

Now summing both sides of (7) from $n=2$ to infinity and using (1.18), we have

$$
\begin{aligned}
c_{p, q}= & (-1)^{q} \sum_{r=0}^{p-2}\left(\begin{array}{c}
q+r-1 \\
q-1
\end{array}\right) c_{p-r, q+r} \\
& +\sum_{r=0}^{q-2}\left(\begin{array}{c}
p+r-1 \\
p-1
\end{array}\right) \zeta(p+r) \zeta(q-r)+\sum_{n=2}^{\infty} C_{n}
\end{aligned}
$$


and

$$
\begin{aligned}
\sum_{n=2}^{\infty} C_{n} & =-(-1)^{q}\left(\begin{array}{c}
p+q-2 \\
p-1
\end{array}\right) \sum_{a=1}^{\infty} \frac{1}{a^{p+q-2}} \sum_{n=a+1}^{\infty} \frac{1}{n(n-a)} \\
& =-(-1)^{q}\left(\begin{array}{c}
p+q-2 \\
p-1
\end{array}\right) \sum_{a=1}^{\infty} \frac{1}{a^{p+q-1}}\left(\frac{1}{a}+\sum_{j=1}^{a-1} \frac{1}{j}\right) \\
& =-(-1)^{q}\left(\begin{array}{c}
p+q-2 \\
p-1
\end{array}\right)\left(\zeta(p+q)+c_{p+q-1,1}\right) .
\end{aligned}
$$

It follows from (8) and (9) that

$$
\begin{aligned}
c_{p, q}= & (-1)^{q} \sum_{r=0}^{p-2}\left(\begin{array}{c}
q+r-1 \\
q-1
\end{array}\right) c_{p-r, q+r} \\
& +\sum_{r=0}^{q-2}(-1)^{r}\left(\begin{array}{c}
p+r-1 \\
p-1
\end{array}\right) \zeta(p+r) \zeta(q-r) \\
& -(-1)^{q}\left(\begin{array}{c}
p+q-2 \\
p-1
\end{array}\right)\left(\zeta(p+q)+c_{p+q-1,1}\right) .
\end{aligned}
$$

Setting $q=1$ in (10), we get

$$
c_{p, 1}=-\sum_{r=2}^{p-1} c_{p-r+1, r}+\zeta(p+1) \quad(p-1 \in \mathbb{N}),
$$

which, for $p=2$, yields a well-known formula:

$$
\sum_{r=1}^{\infty} \frac{H_{r}}{(r+1)^{2}}=\zeta(3),
$$

which was first discovered by Euler in 1775 and has a long history as noted in Shen [7, p. 1392].

From (5) and (11), we have

(13) $\sum_{r=1}^{\infty} \frac{H_{r}}{(r+1)^{p}}=\frac{p}{2} \zeta(p+1)-\frac{1}{2} \sum_{r=2}^{p-1} \zeta(r) \zeta(p-r+1) \quad(p-1 \in \mathbb{N})$,

which is also a well-known formula due, originally, to Euler, and, for $p=2$, immediately reduces to (12) by considering the empty sum to be understood (as usual) to be nil.

Note that Euler's formula (13) has been redeveloped in various ways, since Euler. 


\section{References}

[1] D. Borwein, J. M. Borwein and R. Girgensohn, Explicit evaluation of Euler sums, Proc. Edinburgh Math. Soc. 38 (1995), 277-294.

[2] J. Choi and T. Y. Seo, Formal manipulations of double series and their applications, Pusan-Kyongnam Math. J. 11 (1995), 259-269.

[3] J. Choi, Explicit formulas for Bernoulli polynomials of order n, Indian J. pure appl. Math. 27 (1996), 667-674.

[4] N. Nielsen, Die Gammafunktion, Chelsea Publishing Company, Bronx and New York, 1965.

[5] E. D. Rainville, Special Functions, The Macmillan Company, New York, 1960.

[6] I. J. Schwatt, An Introduction to the Operations with Series (2nd Edi.), Chelsea Publishing Company, New York, 1965.

[7] Li-Chien Shen, Remarks on some integrals and series involving the Stirling numbers and $\zeta(n)$, Trans. Amer. Math. Soc. 347 (1995), 1391-1399.

[8] H. M. Srivastava and J. Choi, Series Associated with the Zeta and Related Functions, Kluwer Academic Publishers, Dordrecht, Boston, and London, 2001.

Department of Mathematics

College of Natural Sciences

Dongguk University

Kyongju 780-714, Korea

E-mail: junesang@mail.dongguk.ac.kr 